\title{
(a) Alterations in the metabolic and cardiorespiratory response to exercise in Huntington's Disease
}

\section{(b) Authors and affiliations}

J Steventon ${ }^{1,2,3}$, J. Collett ${ }^{4,5}$, H. Furby ${ }^{2,3}$, K. Hamana ${ }^{6}$, C. Foster ${ }^{3}$, P. O'Callaghan ${ }^{7}$, A. Dennis ${ }^{8}, R$.

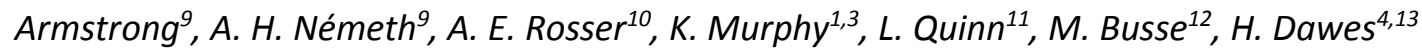

1. School of Physics and Astronomy, Cardiff University

2. NMHRI, School of Medicine, Cardiff University

3. CUBRIC, School of Psychology, Cardiff University

4. Centre for Movement, Occupation and Rehabilitation Sciences, OxINMAHR, Oxford Brookes University, Oxford, UK.

5. Faculty of Health and Life Sciences, Oxford Brookes University, Oxford, UK.

6. School of Healthcare Sciences, Cardiff University

7. Cardiology Department, University Hospital of Wales, Cardiff

8. FMRIB Centre, Nuffield Department of Clinical Neurosciences, John Radcliffe Hospital, University of Oxford, OX3 9DU, UK

9. Nuffield Department of Clinical Neurosciences, University of Oxford, Oxford, UK;

10. IPMCN, School of Medicine, Cardiff University

11. Department of Biobehavioral Sciences, Teachers College, Columbia University, USA.

12. Centre for Trials Research, Cardiff University, UK;

13. Department of Clinical Neurology, University of Oxford, Oxford, UK.

(c) Corresponding author: Dr Jessica Steventon, Cardiff University Brain Research Imaging Centre (CUBRIC), Maindy Road, Cardiff, UK.CF24 4HQ. Email: steventonjj@cardiff.ac.uk Telephone: +44 (0) 2920688758

(d) Word count: 2,994

(e) Running title: An altered exercise response in $H D$

(f) Keywords: Huntington's disease, exercise, physiology, metabolism, cardiorespiratory 
3

4

5

6

7

8

\section{Abstract}

\section{Background}

Limited data suggests that an altered metabolic and cardiorespiratory exercise response may affect exercise performance in individuals with Huntington's disease (HD). There is no clear exploration of the response in individuals at different stages of the disease or in relation to genetic markers. This study aimed to examine the exercise response and recovery of HD participants, and the relationship to genetic and clinical markers.

\section{Method}

HD gene-positive participants ( $n=31 ; 9$ pre-manifest; 22 manifest HD) and a healthy control group $(n=29)$ performed an incremental exercise test until exhaustion. Performance, cardiorespiratory, metabolic and perceptual responses to exercise were determined from a maximal cycle ergometer test throughout the exercise test and during a recovery period.

\section{Results}

During sub-maximal exercise, metabolic (lactate levels, oxygen uptake) and cardiorespiratory markers (heart rate) were elevated in HD participants compared to controls. Lactate elevation was specific to pre-manifest HD participants. Work capacity was reduced in both pre-manifest and manifest HD participants with tests terminated with no difference in metabolic, perceptual or cardiorespiratory markers. Submaximal oxygen uptake was correlated with motor score, whilst peak measures were unrelated to genetic or clinical markers. Heart rate recovery was attenuated in pre-manifest and manifest HD participants.

\section{Conclusions}

Our findings confirm metabolic and cardiorespiratory deficits reduce exercise performance and affect recovery from an early stage in HD, with submaximal deficits related to phenotypic expression. Exercise capacity appears to be limited by an altered movement economy, thus clinicians should consider an altered exercise response and recovery may affect prescription in HD. 


\section{Introduction}

Huntington's disease (HD) is a neurodegenerative disorder caused by the expansion of a polyglutamine stretch within the Huntingtin gene ${ }^{1}$. Neuropathology causes progressive motor disturbances, cognitive dysfunction and psychiatric symptoms ${ }^{2}$. However, as the mutant Huntingtin gene is ubiquitously expressed, people with HD also experience an array of peripheral organ dysfunctions, including a severe metabolic phenotype, weight loss, abnormal fat and glucose homeostasis, cardiomyopathy, skeletal muscle wasting and reduced muscle strength ${ }^{3-6}$.

In healthy people, exercise interventions reduce all-cause mortality and are beneficial for cardiorespiratory fitness, muscle strength, mental health and cognition ${ }^{7-10}$. Exercise prescription has also been shown to be safe, feasible and in some cases, beneficial for motor symptoms in $\mathrm{HD}^{11-13}$. However, results have been variable and detrimental effects of exercise training have also been reported ${ }^{4,14}$. These conflicting and variable findings may reflect an altered physiological and perceptual response to exercise in $\mathrm{HD}^{15}$ which may consequentially affect exercise prescription outcomes. Importantly, the variability in the response to exercise suggests that targeted exercise prescription may be required for people with HD.

In order to develop targeted approaches, it is necessary to understand what factors affect the pathophysiological response to exercise. The current study was designed to improve knowledge on who may benefit from exercise prescription by understanding individual exercise responses. Previous work has found manifest HD participants experience an early increase in blood lactate and a reduced work capacity and peak oxygen consumption, with no abnormalities in peak cardiac and ventilatory performance ${ }^{16}$. Cardiac alterations and differences in perceived exertion were also found at lower exercise intensities in HD participants prior to commencing an exercise intervention ${ }^{15}$, and an increased oxygen cost of exercise has been demonstrated ${ }^{11}$.

Previous studies in HD showing an altered exercise response were measured at a distinct exercise stage, most commonly the response to maximal exercise intensity ${ }^{11,17}$, whilst cardiorespiratory and metabolic recovery after exercise has not been previously studied in HD. Here, we measured the incremental response to exercise across the entirety of a cardiopulmonary test and recovery period. We hypothesised that the physiological responses to the submaximal, maximal and recovery phases may differ between controls and HD participants, which may relate to clinical and genetic disease markers. 
61

62

63

64

65

66

67

68

69

\section{Methods}

\section{Participants}

Thirty-one HD participants (22 manifest, 9 pre-manifest) were recruited from specialist HD clinics in Cardiff, UK and Oxford, UK. Twenty-nine gene-negative controls were matched for age, gender and self-reported physical activity levels. Demographic and clinical data are shown in Table 1. Data was collected with ethical approval and participants gave informed consent.

\section{Exercise protocol}

Participants completed an incremental cycle ergometer exercise test ${ }^{18}$ (Excalibur Sport, Lode, Netherlands) involving two minutes of rest, two minutes of unloaded cycling, followed by 25-watt increments every two minutes, starting at 50-watts (Supplementary Figure 1). The exercise test was symptom limited; individuals pedalled at 50 revolutions/minute until discomfort or fatigue due to effort prevented them from maintaining the work rate. A 10-point Borg Scale measured perceived exertion, fatigue in the legs and dyspnea. At 2 minute intervals, Borg ratings were collected along with whole blood capillary lactate concentration (Lactate pro, UK).

Pulmonary gas exchange was measured on a breath-by-breath basis (MetaMax 3B, Cortex Biophysik GmbH, Leipzig, Germany) and averaged every 30-seconds. Direct measurements of oxygen consumption $\left(\mathrm{O}_{2}\right)$, carbon dioxide production $\left(\mathrm{CO}_{2}\right)$, minute ventilation $(\mathrm{VE})$, and derived variables including the respiratory exchange ratio $\left(\mathrm{RER}\right.$, i.e., $\left.\mathrm{O}_{2} / \mathrm{CO}_{2}\right)$, oxygen pulse $\left(\mathrm{O}_{2} / \mathrm{HR}\right)$ and the ventilatory equivalents for oxygen $\left(\mathrm{VE} / \mathrm{O}_{2}\right)$ and carbon dioxide $\left(\mathrm{VE} / \mathrm{CO}_{2}\right)$ were obtained. Heart rate was continuously recorded using short-range telemetry (Polar S810, Finland).

After reaching exhaustion, participants cycled at an unrestrained speed at 25-watts for 2 minutes. Participants then transferred to a seated position and heart rate, blood pressure, lactate and Borg ratings were recorded every 2-minutes for 10-minutes. Additional protocol details can be found in the supplementary information.

\section{Clinical and genetic measures}

The motor component (TMS) of the UHDRS and the Total Functional Capacity (TFC) ${ }^{19}$ score were recorded. Self-reported physical activity levels were recorded using the International Physical Activity Questionnaire [IPAQ $]^{20}$. CAG repeat length data was available for $19 \mathrm{HD}$ participants. To assess 

Modalities Test (SDMT).

91

92

\section{Statistical analyses}

HD participants were stratified according to disease stage (see Table 1 ; manifest HD participants = TFC score $<13$ and TMS $>18$, Supplementary Figure 2 shows TFC range in manifest group).

As sample size differed across test stages due to different termination points, multiple univariate analyses of variance (ANOVA's) assessed group differences at each test stage (rest, submaximal stages [50-, 75-, 100- and 125-watts], and peak performance), using SPSS software [IBM, version 23]. Multiple comparisons were corrected for using the false discovery rate (FDR) at $p<0.05$. Where results survived the FDR correction, Bonferroni-adjusted post-hoc pairwise analyses examined group differences. Multivariate ANOVA's were conducted for the recovery measures with post-hoc Bonferroni-adjusted analyses.

Where group differences were found, the relationship with clinical and genetic data was tested using a Pearson's correlation analysis.

A slope analysis assessed the relationship between physiology measures and work rate, from rest to peak. The oxygen uptake efficiency slope (OUES) ${ }^{21}$, was determined from rest to peak using the following equation:

$V \dot{O}_{2}=a \log \dot{V E}+b$

Where a represents the OUES, the rate of increase in $\mathrm{VO}_{2}$ in response to VE.

[Table 1 here]

\section{Results}

Despite no significant difference in age, BMI, and IPAQ score between control and HD participants overall (all $p>0.05$ ), manifest HD participants were significantly older than pre-manifest HD and controls participants, thus age was included as a covariate in all analyses.

\section{Exercise protocol compliance}

Four HD participants were excluded from the analysis as they were unable to achieve the required speed of 50 revolutions/min (see Supplementary Table 1). One HD participant experienced syncope following maximal exertion. 
Resting $\mathrm{VO}_{2}$, heart rate, $\mathrm{RER}$, mean arterial blood pressure (MAP) and lactate levels did not different between HD participants and controls ( $p>0.05$, Figure 1). Perceptually, there was no difference in resting ratings of fatigue $(p>0.05)$.

\section{Submaximal exercise function}

Exercise parameters across test stages are shown in Figure 1.

Physiology. $\mathrm{VO}_{2}$ was elevated in manifest $\mathrm{HD}$ participants compared to controls at 50 watts (33\% increase, $\left.F_{2,50}=5.42, p=0.006\right)$ and 75 watts $\left(24.3 \%\right.$ increase, $\left.F_{2,50}=3.45, p=0.035\right)$ with no differences between pre-manifest and control participants $(p>0.05)$. Similarly, heart rate was elevated in manifest HD participants compared to controls at 50 and 75 watts $\left(F_{2,49}=5.54\right.$ and 6.47 respectively, $p<0.05)$. In pre-manifest participants, heart rate was elevated compared to controls at 75 watts only $(p=0.024)$.

There was a main effect of group (HD vs. controls) on lactate levels at 50 watts $\left(F_{1,37}=5.36, p=0.035\right)$, 75 watts $\left(F_{1,35}=8.21, p=0.028\right)$ and 100 watts $\left(F_{1,34}=5.90, p=0.034\right)$. Lactate was higher in premanifest HD participants compare to controls, surviving Bonferroni correction at 75 and 100 watts ( $p$ $=0.014$ and 0.039 ) but not 50 watts (controls vs. manifest $\mathrm{HD}, \mathrm{p}=0.053$ ). The difference between controls and manifest HD participants was significant at 50 and 75 watts $(p=0.027$ and 0.049 respectively) before age was accounted for, however with age as a covariate, this difference was not significant.

Results from the slope analysis are shown in Supplementary Table 2. There was no difference in the OUES nor the relationship between work rate and $\mathrm{VO}_{2}$, heart rate and lactate between control and $\mathrm{HD}$ participants (all $p>0.05$ ).

Perceptual responses. There were no group difference in perceived exertion ratings at any workload between 50 and 125 watts $(p>0.05)$. 
149 Figure 2 shows the individual and group data at exhaustion. Age-adjusted marginal means are shown 150 in Supplementary Table 3.

151 Performance. Volitional exhaustion occurred at a lower working capacity (Wpeak) in HD participants $152\left(F_{1,53}=11.26, p=0.001\right.$, Figure $\left.2 A\right)$. Pre-manifest and manifest participants had a lower Wpeak 153 compared to controls ( $p=0.049$ and 0.026 respectively); there was no difference in Wpeak between 154 pre-manifest and manifest HD participants $(p>0.05)$.

155 Physiology. There were no differences in $\dot{\mathrm{V}} \mathrm{O}_{2}$ peak, heart rate, oxygen pulse, RER, VE, VE/VCO 156 (controls $=28.9 \pm 0.8 ;$ pre-manifest $=30.7 \pm 1.4 ;$ manifest $\mathrm{HD}=30.0 \pm 1.0$ ), $\mathrm{VE} / \mathrm{O}_{2}$ (controls $=35.5 \pm$ 157 1.4; pre-manifest $=33.2 \pm 2.1$; manifest $\mathrm{HD}=34.9 \pm 1.6$ ) at exhaustion between $\mathrm{HD}$ and control 158 participants $(p>0.05)$.

159 Blood lactate production was $25.3 \%$ lower in HD participants at exhaustion compared to controls $\left(\mathrm{F}_{1,40}\right.$ $160=9.37, p=0.004$, Figure $2 \mathrm{E})$ and was lower in manifest HD participants compared to controls $(p=$ 161 0.032). Pre-manifest HD participants did not differ from controls. To determine the effect of Wpeak on lactate production, peak lactate levels were normalised by Wpeak. There was no difference in normalised peak lactate between control and HD participants (controls $=0.057 \pm 0.003$; pre-manifest $H D=0.060 \pm 0.004$; manifest $H D=0.050 \pm 0.003, p>0.05$ ).

Perceptual responses. There were no differences in perceived exertion ratings between HD participants and control participants at volitional exhaustion $(p>0.05)$.

\section{Exercise recovery}

169 Physiology. On a group level, heart rate did not differ between HD and control participants (Figure $1703 \mathrm{~A})$. To examine individual variability in heart rate recovery (HRR), the change in heart rate from peak 171 was calculated. HRR was slower in HD participants compared to controls $\left(F_{10,70}=1.98, p<0.05\right.$, Figure 172 3B). HRR was higher in controls compared to manifest HD participants at 2-minutes post exercise $(p=$ 173 0.011), whereas after 4-minutes, HRR was higher in controls compared to pre-manifest and manifest 174 participants ( $p=0.038$ and 0.007 respectively). At 6-, and 8-minutes post exercise, HRR remained 175 higher in controls compared to pre-manifest participants ( $p=0.016$ and 0.021 respectively). There 
were no differences in HRR between manifest and pre-manifest HD participants. Ten minutes following exercise, the difference between HRR in pre-manifest participants and controls was not significant $(p=0.07)$.

Lactate levels were reduced in manifest HD participants compared to controls at 2- and 4-minutes post exercise ( $p=0.012$ and 0.034 respectively, Figure $3 C$ ), with no differences between control and pre-manifest HD participants, and pre-manifest and manifest HD participants. To account for peak lactate levels, lactate change (from peak) was calculated. There were no differences between control and HD participants in lactate change during recovery ( $p>0.05$, Figure 3D).

Perceptual response. Self-reported ratings of fatigue during the recovery phase did not differ between HD and control participants (Figure 3, p > 0.05).

\section{Relationship between exercise measures and genetic and phenotypic HD expression}

UHDRS TMS, where a higher score indicates greater movement disorder, was correlated with $\mathrm{VO}_{2}$ at 50 watts $(r=0.49, p=0.032)$, and 75 watts $(r=0.44, p=0.041$, Supplementary Figure 3$)$. There was no correlation between submaximal $\mathrm{VO}_{2}$ and UHDRS TFC or CAG repeat length (all $p>0.05$ ). Heart rate at 50- and 75-watts, and lactate levels at 50-, 75- and 100-watts were not correlated with cUHDRS TMS and TFC or CAG repeat length, all $p>0.05$.

No correlations were found between clinical data (UHDRS TMS and TFC), CAG repeat length and peak lactate or Wpeak, all $p>0.05$.

The correlation between HRR at 4- and 6-minutes post exercise and the UHDRS TMS ( $r=-0.40$ and $0.40, p=0.039$ and 0.041 uncorrected) and UHDRS TFC ( $r=0.38$ and $0.46, p=0.053$ and 0.017 uncorrected) was not significant after multiple comparison adjustments.

This study shows an altered exercise response in people with HD during a graded exercise test. Despite normal resting physiology, upon initiation of low intensity exercise, heart rate, lactate, and oxygen uptake were elevated in HD participants compared to controls, suggestive of a movement economy 
deficit and altered metabolism. The early elevation in lactate was observed in pre-manifest HD participants specifically, suggesting that abnormal oxidative metabolism in skeletal muscle may be an early feature of HD prior to functional decline. In contrast, elevated oxygen uptake at low intensities was related to greater motor dysfunction in HD participants. In agreement with previous work ${ }^{16}$, volitional exhaustion occurred at a lower working capacity in HD participants and both pre-manifest and manifest HD participants produced less lactate at maximal effort. Almost one fifth of manifest HD participants were unable to maintain the required cycling speed, highlighting an altered exercise tolerance and physical ability in HD.

The altered cardiorespiratory and metabolic response to exercise may account for the variable responses to exercise prescription previously reported in $\mathrm{HD}^{17}$. The relationship between oxygen uptake during submaximal exercise and motor functioning suggest some of the variability can be accounted for by phenotypic disease expression, however metabolic differences were not accounted for by genetic load or phenotype, and were evident in pre-manifest HD participants, suggesting a complex physiological response to exercise in HD.

Notably, these novel findings provide support for individualised exercise prescription, and suggest that the effect of long-term exercise training may vary between individuals based on the prescribed exercise intensity and their subsequent physiological response. Further studies measuring the response to exercise are therefore recommended throughout the prescription period in order to understand if disturbances in exercise response interact with exercise prescription outcomes. In addition, it would be valuable to measure creatine kinase following exercise to explore muscle cell disturbance in $\mathrm{HD}$ and to further explore autonomic functioning.

Whereas previous work has shown a reduced $\dot{\mathrm{V}}_{2}$ peak in manifest $\mathrm{HD}^{16}$, we failed to replicate this. The discrepancy is most likely explained by the inclusion of age as a covariate in this study; when $\mathrm{VO}_{2}$ was measured as a percent of theoretical maximum capacity according to age, body type, and sex, differences due to HD were no longer observed ${ }^{16}$. In line with previous work ${ }^{16}$, HD participants had a normal ventilatory response to maximal exercise and both clinical data and genetic data did not predict peak measures. CAG repeat length was also not predictive of the submaximal physiological response, suggesting that genetic markers are insensitive to exercise response in $\mathrm{HD}$. The rate of $\mathrm{VO}_{2}$ may be affected by how effectively oxygen is delivered to the muscles (respiratory and cardiovascular systems), how it is utilised (metabolic systems) and how hard the muscles are working (neuromuscular systems). We propose that higher $\mathrm{VO}_{2}$ at submaximal exercise and not at peak may be due to muscles working harder in people with HD due to chorea and/or poor co-ordination which requires greater 
oxygen use. At peak exercise, aerobic metabolism is limited due to issues with bioenergetics and mitochondrial functioning in the HD group.

After reaching exhaustion, heart rate recovery was attenuated in HD participants. This is a novel finding; impaired heart rate recovery is an independent predictor of mortality in healthy populations ${ }^{22}$. The fall in heart rate immediately after exercise is a function of the reactivation of the parasympathetic nervous system, mediated by vagal reactivation in the first two minutes of recovery ${ }^{23}$. It is not clear whether vagal reactivation continues to mediate heart rate recovery for the eight minutes observed here, or whether other mechanisms drive the slowed response. Due to the cool-down protocol used, it is not possible to compare heart rate recovery with normative data attained immediately after exhaustive exercise cessation, however our findings complement work showing reduced cardiovagal modulation in middle-stage $\mathrm{HD}^{24,25}$. The elevated heart rate observed during submaximal exercise in HD participants replicates Dawes et al. ${ }^{15}$ and increases the robustness of the observation that people with HD do not reach steady heart rates when initiating low-intensity exercise. This is informative for exercise prescription; applying objective submaximal markers as opposed to training intensities based on maximal measures (e.g. heart rate, $\mathrm{VO}_{2}$ ), and measuring exercise recovery as an indicator of intervention effectiveness may be more appropriate.

The difference observed in peak lactate is most likely driven by differences in task performance. Working capacity was reduced in HD participants, and when peak lactate was normalised for maximal work rate, the lactate differences were no longer significant. This suggests that HD participants were unable to work as hard on the exercise test which contributed to the lower lactate levels and is supported by previous work showing a reduced work capacity in manifest participants ${ }^{16}$. The reduced working capacity in pre-manifest HD participants suggests an underlying energy deficit rather than reduced muscle bulk.

Altered metabolism was also evident during submaximal exercise. The failure of oxidative mechanisms can affect lactate production and clearance and previous work has shown elevated lactate production in symptomatic HD participants during a cardiopulmonary test at 50 watts and 75 watts ${ }^{16}$. We replicated this finding, however, lactate was also significantly higher in pre-manifest HD participants compare to controls at 75 and 100 watts, whereas the difference between controls and manifest HD participants was not significant after accounting for age. The discrepancy in the results may be partly explained by higher fitness levels in the current cohort and a broader symptomatic HD group.

A common limitation in clinical exercise research is the physiological effect of medication. Participants in this study were prescribed a diverse range of medications which alter metabolic 
pathways ${ }^{26}$. It is not known if observed metabolic and cardiac deficits are a cause or consequence of HD toxicity, and/or mediated by medication. A comprehensive large-scale study is required to unpick medication effects, with the metabolic consequences of drugs in the same sub-class varying substantially due to differences in receptor pharmacology. Metabolic deficits observed in animal models and cell cultures of HD suggest that observed effects are not purely driven by medication ${ }^{27,28}$, however they may account for some variability in exercise prescription responses.

Overall, we have demonstrated that metabolic and cardiorespiratory deficits contribute towards a reduced exercise performance and affects recovery in HD. Autonomic dysfunction has been reported in a variety of neurodegenerative dementias 29,30 and further work is required to elucidate whether deficits are a direct consequence of peripherally expressed mutant huntingtin protein, or secondary to a general decline in health or neurological dysfunction. This knowledge will be crucial for targeted exercise prescription in $\mathrm{HD}$ and for determining outcome measures.

\section{Acknowledgments}

We gratefully acknowledge the participants in this trial, as well as their family members and caregivers. We would also like to acknowledge the Waterloo Foundation, the Gossweiler Foundation, the Wellcome Trust (200804/Z/16/Z), the Neuroscience and Mental Health Research Institute (Cardiff University, UK); the NIHR Oxford Biomedical Research Centre (BRC) (UK) and the Oxford Health BRC for funding this work.

\section{References}

1. Gusella, J. F., MacDonald, M. E., Ambrose, C. M. \& Duyao, M. P. Molecular genetics of Huntington's disease. Arch. Neurol. 50, 1157-63 (1993).

2. Bates, G., Harper, P. S. \& Jones, L. Huntington's disease. (New York: Oxford University Press, 2002).

3. Busse, M. E., Hughes, G., Wiles, C. M. \& Rosser, A. E. Use of hand-held dynamometry in the evaluation of lower limb muscle strength in people with Huntington's disease. J. Neurol. 255, 1534-1540 (2008).

4. Kosinski, C. M. et al. Myopathy as a first symptom of Huntington's disease in a Marathon runner. Mov. Disord. 22, 1637-1640 (2007).

5. Lodi, R. et al. Abnormal in vivo skeletal muscle energy metabolism in Huntington's disease and dentatorubropallidoluysian atrophy. Ann. Neurol. 48, 72-6 (2000).

6. Djoussé, L. et al. Weight loss in early stage of Huntington's disease. Neurology 59, 1325-30 (2002). 
7. Colcombe, S. \& Kramer, A. F. FITNESS EFFECTS ON THE COGNITIVE FUNCTION OF OLDER ADULTS: A Meta-Analytic Study. 14, (2003).

8. Cooney, G. M. et al. Exercise for depression. Cochrane database Syst. Rev. CD004366 (2013). doi:10.1002/14651858.CD004366.pub6

9. Arem, H. et al. Leisure Time Physical Activity and Mortality. JAMA Intern. Med. 175, 959 (2015).

10. King, N. A., Hopkins, M., Caudwell, P., Stubbs, R. J. \& Blundell, J. E. Beneficial effects of exercise: shifting the focus from body weight to other markers of health. Br. J. Sports Med. 43, 924-927 (2009).

11. Frese, S. et al. Exercise effects in Huntington disease. J. Neurol. (2016). doi:10.1007/s00415016-8310-1

12. Khalil, H. et al. What effect does a structured home-based exercise programme have on people with Huntington's disease? A randomized, controlled pilot study. Clin Rehabil 27, 646658 (2013).

13. Quinn, L. et al. A randomized, controlled trial of a multi-modal exercise intervention in Huntington's disease. Parkinsonism Relat. Disord. 31, 46-52 (2016).

14. Potter, M. C., Yuan, C., Ottenritter, C., Mughal, M. \& van Praag, H. Exercise is not beneficial and may accelerate symptom onset in a mouse model of Huntington's disease. PLoS Curr. 2, RRN1201 (2010).

15. Dawes, H. et al. Exercise testing and training in people with Huntington's disease. Clin. Rehabil. 29, 196-206 (2015).

16. Ciammola, A. et al. Low anaerobic threshold and increased skeletal muscle lactate production in subjects with Huntington's disease. Mov. Disord. 26, 130-7 (2011).

17. Dawes, H. et al. Exercise testing and training in people with Huntington's disease. Clin. Rehabil. 0269215514540921- (2014). doi:10.1177/0269215514540921

18. Collett, J. et al. Exercise for multiple sclerosis: a single-blind randomized trial comparing three exercise intensities. Mult. Scler. J. 17, 594-603 (2011).

19. Shoulson, I. \& Fahn, S. Huntington disease: clinical care and evaluation. Neurology 29, 1-3 (1979).

20. Craig, C. L. et al. International physical activity questionnaire: 12 -country reliability and validity. Med. Sci. Sports Exerc. 35, 1381-95 (2003).

21. Baba, R. et al. Oxygen uptake efficiency slope: a new index of cardiorespiratory functional reserve derived from the relation between oxygen uptake and minute ventilation during incremental exercise. J. Am. Coll. Cardiol. 28, 1567-72 (1996).

22. Cole, C. R., Blackstone, E. H., Pashkow, F. J., Snader, C. E. \& Lauer, M. S. Heart-Rate Recovery Immediately after Exercise as a Predictor of Mortality. N. Engl. J. Med. 341, 1351-1357 (1999).

23. Imai, K. et al. Vagally mediated heart rate recovery after exercise is accelerated in athletes but blunted in patients with chronic heart failure. J. Am. Coll. Cardiol. 24, 1529-35 (1994).

24. Andrich, J. et al. Autonomic nervous system function in Huntington's disease. J. Neurol. Neurosurg. Psychiatry 72, 726-31 (2002). 
25. Bar, K. J. et al. Cardiovagal modulation upon postural change is altered in Huntingtons disease. Eur. J. Neurol. 15, 869-871 (2008).

26. Reynolds, G. P. \& Kirk, S. L. Metabolic side effects of antipsychotic drug treatment pharmacological mechanisms. Pharmacol. Ther. 125, 169-179 (2010).

27. Tsang, T. M. et al. Metabolic Characterization of the R6/2 Transgenic Mouse Model of Huntington's Disease by High-Resolution MAS ${ }^{1}$ H NMR Spectroscopy. J. Proteome Res. 5, 483-492 (2006).

28. Naia, L. et al. Insulin and IGF-1 regularize energy metabolites in neural cells expressing fulllength mutant huntingtin. Neuropeptides 58, 73-81 (2016).

29. Goldstein, D. S. Dysautonomia in Parkinson's disease: neurocardiological abnormalities. Lancet. Neurol. 2, 669-76 (2003).

30. Chaudhuri, K. R. Autonomic dysfunction in movement disorders. Curr. Opin. Neurol. 14, 50511 (2001).

\section{Figure Legends}

Figure 1 Response to submaximal exercise. Results are marginal means adjusted for age, error bars = standard errors of the mean. RER: Respiratory exchange rate. RPE: Ratings of perceived exertion. ${ }^{*} p<0.05$ Bonferroni-adjusted. + unadjusted $p<$ 0.05. preHD: pre-manifest, HD: manifest HD.

Figure 2 Peak exercise response at test termination. Black line represents mean; grey box depicts $95 \% \mathrm{Cl}$. RER: respiratory exchange ratio $\left(\mathrm{VCO}_{2}: \mathrm{VO}_{2}\right)$; RPE: Rating of perceived exertion. PreHD: pre-manifest HD participants. HD: Manifest HD participants. P-values are Bonferroni- adjusted.

Figure 3 Recovery response $[B] \&[D]$ show absolute change from peak. Results are marginal means adjusted for age. Error bars $=$ standard errors of the mean. RPE: rating of perceived exertion. HR: heart rate (beats/minute). ${ }^{*} p<0.05, * * p<0.01$. 